\title{
REVIEW ARTICLE \\ Part I. Analysis of data gaps pertaining to Salmonella enterica serotype Typhi infections in low and medium human development index countries, 1984-2005
}

\author{
J. A. CRUMP ${ }^{1 *}$, P. K. RAM ${ }^{1}$, S. K. GUPTA ${ }^{1}$, M. A. MILLER ${ }^{2}$ AND E. D. MINTZ ${ }^{1}$ \\ ${ }^{1}$ Enteric Diseases Epidemiology Branch, National Center for Zoonotic, Vectorborne, and Enteric Diseases, \\ Centers for Disease Control and Prevention, Atlanta, GA, USA \\ ${ }^{2}$ Fogarty International Center, National Institutes of Health, Bethesda, MD, USA
}

(Accepted 30 June 2007; first published online 9 August 2007)

\section{SUMMARY}

There are only 10 contemporary, population-based studies of typhoid fever that evaluate disease incidence using blood culture for confirmation of cases. Reported incidence ranged from 13 to $976 / 100000$ persons per year. These studies are likely to have been done preferentially in high- incidence sites which makes generalization of data difficult. Only five of these studies reported mortality. Of these the median (range) mortality was $0 \%(0-1 \cdot 8 \%)$. Since study conditions usually involved enhanced clinical management of patients and the studies were not designed to evaluate mortality as an outcome, their usefulness for generalizing case-fatality rates is uncertain. No contemporary population-based studies reported rates of complications. Hospitalbased typhoid fever studies reported median (range) complication rates of $2 \cdot 8 \%(0 \cdot 6-4.9 \%)$ for intestinal perforation and case-fatality rates of $2 \cdot 0 \%(0-14 \cdot 8 \%)$. Rates of complications other than intestinal perforation were not reported in contemporary hospital-based studies. Hospitalbased studies capture information on the most severe illnesses among persons who have access to health-care services limiting their generalizability. Only two studies have informed the current understanding of typhoid fever age distribution curves. Extrapolation from population-based studies suggests that most typhoid fever occurs among young children in Asia. To reduce gaps in the current understanding of typhoid fever incidence, complications, and case-fatality rate, large population-based studies using blood culture confirmation of cases are needed in representative sites, especially in low and medium human development index countries outside Asia.

\section{INTRODUCTION}

The global incidence of typhoid fever was estimated to be about 21650000 illnesses in 2000 and varies greatly between regions. Typhoid fever incidence is

* Author for correspondence: J. A. Crump, MB, ChB, DTM\&H, Medical Epidemiologist, Enteric Diseases Epidemiology Branch, National Center for Zoonotic, Vectorborne, and Enteric Diseases, Centers for Disease Control and Prevention, Atlanta, GA 30333, USA.

(Email: jcrump@cdc.gov)

This is the first of three papers, appearing in successive issues of the Journal, reviewing the analysis of data gaps pertaining to infections in low and medium human development index (HDI) countries. highest among infants and children living in South Central and South East Asia [1]. Typhoid fever is a severe systemic illness that is characterized by sustained fever, systemic toxicity and abdominal pain [2]. S. Typhi colonizes only humans. The disease is acquired by consuming water or food contaminated by the faeces of a person who has typhoid fever or who is a carrier [3]. While transmission via sexual relations between men has also been reported [4], true person-to-person transmission is rare. The $\mathrm{ID}_{50}$ of $S$. Typhi is about $10^{6}$ organisms by ingestion, but varies considerably with gastric acidity. The risk of 
Table 1. Terms used in literature search to identify gaps in data on enteric disease burden

\begin{tabular}{l}
\hline \hline Burden of disease \\
Epidemiology \\
Morbidity \\
Mortality \\
Disease outbreaks \\
Incidence \\
Prevalence \\
Seasons \\
Population surveillance \\
Age distribution \\
Longitudinal survey \\
Pathogen-specific terms for typhoid fever \\
Typhoid \\
Typhoid fever \\
Enteric fever \\
Salmonella Typhi \\
\hline \hline
\end{tabular}

infection increases as the size of the inocula increases [5].

Salmonella enterica subspecies enterica serotype Typhi (Salmonella Typhi) belong to the family of Enterobacteriaceae and are Gram-negative, motile, non-lactose-fermenting bacilli. Although most S. enterica serotypes cannot be distinguished by biochemical reactions, $S$. Typhi can be provisionally identified by its production of only trace amounts of hydrogen sulphide and being less active biochemically than other serotypes within the subspecies. Confirmation depends on the detection of surface antigens using specific antisera. $S$. Typhi belongs to Salmonella serogroup D and possesses somatic antigen O9, a single flagellar antigen $\mathrm{Hd}$, and the polysaccharide virulence antigen Vi. Resistance to traditional first-line antimicrobial agents, such as ampicillin, chloramphenicol, and trimethoprim-sulfonamide combinations has emerged worldwide. More recently isolates with reduced susceptibility to fluoroquinolones and third-generation cephalosporins (i.e. ceftriaxone) have been increasingly recovered [6].

Building on our previously published work on typhoid fever epidemiology [1], we conducted a review of the recent scientific literature to identify contemporary gaps in the understanding of the global epidemiology of typhoid fever. An improved understanding of typhoid fever epidemiology would allow policy-makers to decide how to allocate resources to typhoid fever vs. other health priorities and would allow health officials to select and monitor the effectiveness of public health interventions. We summarize below recent available data on the morbidity and mortality burden, the age, and the geographic and temporal distribution of typhoid fever. To provide context to the global epidemiology of typhoid fever, we also describe typhoid diagnostics and pathogen-specific preventive measures. Finally, data gaps and existing research needs are discussed.

\section{METHODS}

We systematically searched the English-language scientific literature published between 1984 and 2005 using the Medline database, restricting the search to low and medium human development countries according to the United Nations Development Programme's Human Development Index (HDI) (http:// hdr.undp.org 2004; accessed 21 March 2005). A set of articles including the relevant epidemiological terms were cross-linked with a set of articles including the relevant pathogen-specific terms (Table 1). The resulting cross-linked set was reviewed for publications addressing typhoid fever morbidity, mortality, age distribution, geographic distribution, temporal distribution, pathogen-specific preventive measures, and diagnostics. Particularly for morbidity and mortality burden, population-based studies with culture confirmation of cases were considered primary data sources. When these were limited, hospital- and clinicbased studies were included. Publications were then evaluated for their contribution to an understanding of the global epidemiology of typhoid fever, and gaps in the data were identified.

Crude incidence figures are given, without correction for sensitivity of blood culture, or for the proportion of febrile episodes for which blood-culture specimens were not collected. Publications were sought with information on the incidence of the infection by region according to the 21 regions of the United Nations Department of Social and Economic Affairs, Population Division (http://esa.un.org/unpp/ index.asp?panel $=5 ; 2004$ revision, accessed 4 October 2005). We examined the correlation between the incidence of typhoid fever and the national per capita gross domestic product (GDP), adjusted for purchasing power parity (PPP) in year 2000 dollars, at the time that the studies were conducted (http:// unstats.un.org/unsd/cdb/cdb_series_xrxx.asp?series_ code $=29922$; accessed 28 July 2006). We searched for publications with information on the age-specific incidence, morbidity, and mortality. 


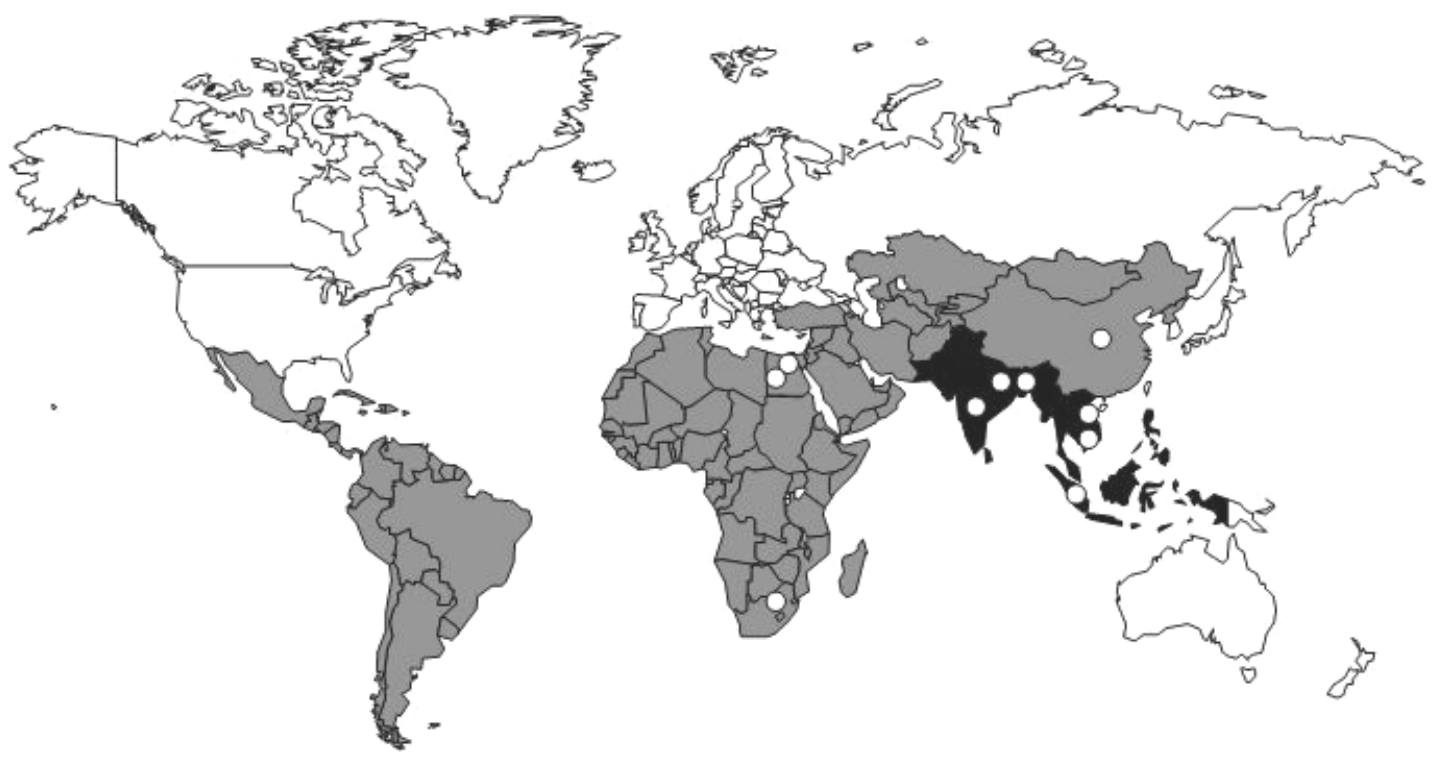

Fig. 1. Geographic distribution of population-based studies of typhoid fever incidence. (Adapted from Crump et al. [1].) $\boldsymbol{\square}$, High incidence ( $>100$ episodes/100 000 per year); $\square$, Medium incidence (10-100 episodes/100 000 per year); $\square$, Low incidence ( $<10$ episodes/100 000 per year); $\square$, region with human development index (HDI) countries; $\bigcirc$, site of contributing disease incidence study.

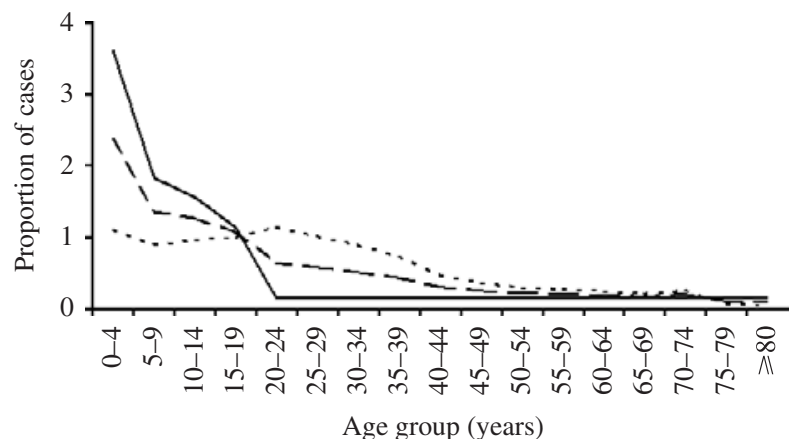

Fig. 2. Age-specific incidence of typhoid fever (from Crump et al. [1]). - High (>100/100000 per year); ---, medium $(10-100 / 100000$ per year); ---, Low $(<10 / 100000$ per year).

\section{RESULTS}

\section{Morbidity}

\section{Incidence}

The most recent published estimate of the global burden of typhoid fever determined that 21650000 illnesses occurred worldwide in 2000 [1] and that the incidence was highest in South and South East Asia and among infants and young children (Figs 1 and 2).

A computer search of the English scientific literature published between 1984 and 2005 from low- and medium-HDI countries for population-based typhoid fever incidence studies using blood culture confirmation of cases yielded 10 publications (Table 2); three studies from Africa (South Africa and Egypt) and seven from Asia (Bangladesh, China, India, Indonesia, Nepal, and Vietnam). Five (50\%) of these publications were reports of vaccine studies and all 10 were from medium-HDI countries. Crude typhoid fever incidence in these studies ranged from 13 to 976 illnesses/100 000 persons per year. Five studies included subjects of all ages; the remainder were restricted to persons within specific age ranges. Because of limitations of blood culture sensitivity for typhoid fever (Table 3) and variation of typhoid fever incidence with age (Fig. 2), it would be appropriate to adjust crude incidence for these factors when attempting to extrapolate typhoid fever incidence to the global population [1].

Among the 10 population-based studies, there was a non-significant relationship between typhoid fever incidence and per capita GDP (Fig. 3) $(R=-0 \cdot 04$, $P=0.9115)$. One study that showed high typhoid incidence was done in South Africa, a country with a relatively high GDP but with considerable maldistribution of wealth. When this outlier study was excluded, a non-significant trend towards an inverse relationship between typhoid fever incidence and per capita GDP is seen $(R=-0.63, P=0.0717)$.

\section{Complications}

Intestinal perforation is an important complication of typhoid fever. Unfortunately, no population-based 
Table 2. Population-based studies of typhoid fever incidence 1984-2005

\begin{tabular}{|c|c|c|c|c|c|c|c|c|}
\hline \multicolumn{3}{|c|}{ Location } & \multirow[b]{2}{*}{ HDI } & \multirow[b]{2}{*}{ Year(s) } & \multirow{2}{*}{$\begin{array}{l}\text { Age } \\
\text { range } \\
(\mathrm{yr})\end{array}$} & \multirow{2}{*}{$\begin{array}{l}\text { Crude } \\
\text { incidence* }\end{array}$} & \multirow{2}{*}{$\begin{array}{l}\text { CFR } \\
(\%)\end{array}$} & \multirow[b]{2}{*}{ Ref. } \\
\hline Area & Region & Country & & & & & & \\
\hline Africa & N Africa & Egypt & Medium & 2001 & All & 13 & n.r. & [7] \\
\hline Africa & N Africa & Egypt & Medium & 2002 & All & 59 & n.r. & [45] \\
\hline Africa & S Africa & South Africa & Medium & $1985-88$ & $5-15$ & 845 & n.r. & {$[28,46]$} \\
\hline Asia & E Asia & China & Medium & 1995-96 & $3-50$ & 21 & n.r. & [37] \\
\hline Asia & SC Asia & India & Medium & 1995-96 & All & 976 & 0 & [14] \\
\hline Asia & SC Asia & Nepal & Medium & $1986-87$ & $5-44$ & 654 & $1 \cdot 8$ & [27] \\
\hline Asia & SC Asia & Bangladesh & Medium & $2000-01$ & All & 390 & 0 & [15] \\
\hline Asia & SC Asia & Indonesia & Medium & $1986-89$ & $3-44$ & 810 & n.r. & [47] \\
\hline Asia & SC Asia & Vietnam & Medium & 1995-96 & All & 198 & 0 & [24] \\
\hline Asia & SC Asia & Vietnam & Medium & $1998-2000$ & $2-4$ & 931 & 0 & [31] \\
\hline
\end{tabular}

HDI, Human development index; CFR, case-fatality rate; n.r., not reported.

* Per 100000 persons per year.

Studies excluded for high human development index country: 2 from Chile [48, 49].

Studies excluded for pre-1984: 1 from Chile [50], 2 from Egypt [51, 52]; 1 from India [53], 1 from Poland [54], 4 from the USSR [55-58], 2 from Yugoslavia [59, 60], 1 from Guyana [61], and 1 from Tonga [62].

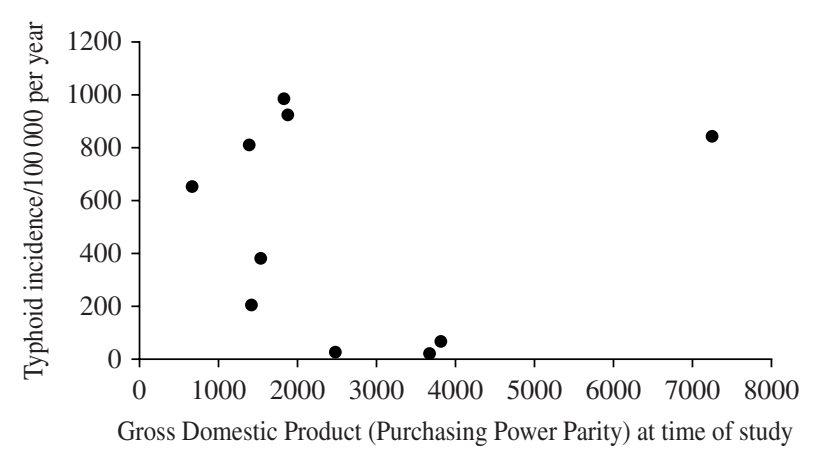

Fig. 3. Incidence of typhoid fever by per capita gross domestic product (adjusted for purchasing power) of low- and medium-HDI countries, 1984-2005 ( $n=10$ studies).

studies that use blood culture confirmation of $S$. Typhi infection report the rates of this or other complications. Hospital-based studies capture information on the most severe illnesses among persons who have access to health-care services and are likely to overestimate the true complication rate substantially [7]. A review of intestinal perforation rates in typhoid fever series from all countries from the preantibiotic era before 1950 indicate median (range) rates of $1.9 \%(0 \cdot 8-6 \cdot 3 \%)$. The same review shows a rate of intestinal perforation in developed countries of $0 \%(0-0 \%)$ in the post-antibiotic era after 1950 and in developing countries in the post-antibiotic era after 1950 of $3 \cdot 6 \%(0 \cdot 1-41 \%)$ [8]. Case-series published between 1984 and 2005 from low- and mediumHDI countries report median (range) intestinal perforation rates of $2 \cdot 8 \%(0 \cdot 6-4 \cdot 9 \%)$ (Table 4$)$. Risk factors for intestinal perforation in typhoid fever include male gender, older age, inadequate antimicrobial therapy, and short duration of symptoms [8-10].

Other complications occur, but their rates are not well quantified in either population- or hospital-based studies. A recent review of typhoid fever complications involving specific organ systems indicated that they occur with the following frequencies in hospital series: central nervous system (encephalopathy, cerebral oedema, subdural empyema, cerebral abscess, meningitis, ventriculitis, transient Parkinsonism, motor neuron disorders, ataxia, seizures, GuillainBarré syndrome, psychosis) 3-35\%; cardiovascular system (endocarditis, myocarditis, pericarditis, arteritis, congestive cardiac failure) $1-5 \%$; pulmonary system (pneumonia, empyema, bronchpulmonary fistula) $1-6 \%$; bone and joint (osteomyelitis, septic arthritis) $<1 \%$; hepatobiliary system (cholecystitis, hepatic abscess, splenic abscess, peritonitis, paralytic ileus); $1-26 \%$, and the genitourinary system (urinary tract infection, renal abscess, pelvic infection, testicular abscess, prostatitis, epididymitis) $<1 \%$. Soft tissue infections (psoas abscess, gluteal abscess, cutaneous vasculitis) and haematological complications (haemophagocytic syndrome) are rare [11].

While the geographic distribution of typhoid fever and HIV overlap, no studies have comprehensively evaluated the effect of HIV on typhoid fever incidence or complications. 
Table 3. Comparison of diagnostic tests for typhoid fever

\begin{tabular}{llllcl}
\hline \hline Assay name & Gold standard & Assay type & Sensitivity & Specificity & Ref. \\
\hline Bone marrow culture & Bone marrow & Culture & $0 \cdot 90^{*}$ & $1 \cdot 0$ & {$[40]$} \\
Blood culture & Bone marrow & Culture & $0 \cdot 40^{*}$ & $1 \cdot 0$ & {$[40]$} \\
Stool culture & Bone marrow & Culture & $0 \cdot 37$ & $<\cdot 0 \dagger$ & {$[40]$} \\
Urine culture & Bone marrow & Culture & $0 \cdot 07$ & $1 \cdot 0$ & {$[40]$} \\
Rose spot culture & Bone marrow & Culture & $0 \cdot 63$ & $1 \cdot 0$ & {$[40]$} \\
Widal test & Blood culture & Antibody detection & $0 \cdot 64$ & $0 \cdot 76$ & {$[44]$} \\
Multi-Test Dip-S-Ticks & Blood culture & Antibody detection & $0 \cdot 89$ & $0 \cdot 53$ & {$[44]$} \\
TyphiDot & Blood culture & Antibody detection & $0 \cdot 79$ & $0 \cdot 89$ & {$[44]$} \\
TUBEX & Blood culture & Antibody detection & $0 \cdot 78$ & $0 \cdot 89$ & {$[44]$} \\
\hline \hline
\end{tabular}

* Varies according to sample volume.

$\dagger$ Varies according to carrier prevalence in study population.

\section{Epidemic vs. endemic disease}

Large typhoid fever outbreaks make substantial contributions to the local disease burden. For example, during a large waterborne outbreak of typhoid fever in Tajiskistan during 1997 more than 1200 cases of typhoid fever were detected each week during the epidemic's peak in the city of Dushanbe which had a population of about 600000 people [12]. While large outbreaks occur, endemic typhoid fever is thought to be responsible for most typhoid fever illnesses worldwide. However, we were unable to find populationbased typhoid fever surveillance studies of sufficient duration to capture incidence before, during, and after typhoid fever epidemics. Therefore, the relative contribution of epidemics to disease burden is not known.

\section{Mortality}

Five (50\%) of 10 population-based studies conducted between 1984 and 2005 and representing 64338 person-years of follow-up report mortality data. These five studies showed median (range) case-fatality rates (CFRs) of $0 \%(0-1.8 \%)$ (Table 2). There are several limitations to generalizing CFRs for typhoid fever from these studies. In all studies ethical requirements governing human subject research would have optimized the clinical management of patients found to have typhoid fever probably leading to lower CFRs than may be seen in the source population. Second, these studies were not designed to assess mortality as a primary outcome.

Hospital-based series provide another way to estimate typhoid fever mortality, but only capture information on the most severe illnesses among persons who have access to health-care services [7]. The literature search identified 28 hospital-based studies reporting CFRs (Table 5): four studies from Africa, 22 from Asia, and one each from Latin America/ Caribbean and Oceania. The median (range) CFR in these studies was $2 \cdot 0 \%(0-14 \cdot 8 \%)$.

A review of published typhoid fever series from the pre-antibiotic era before 1950 showed median (range) CFRs of $11 \%(7 \cdot 5-19 \%)$. The same review shows a median (range) CFR for typhoid fever in developing countries in the post-antibiotic era after 1950 of $6.1 \%$ $(0 \cdot 1-41 \%)[8]$.

Appropriate antimicrobial therapy is known to reduce mortality from typhoid fever [13]. A consequence of the emergence of antimicrobial resistance in $S$. Typhi is that empiric treatment choices for typhoid fever often include antimicrobial agents to which the infecting organism is resistant. This is especially challenging in settings where laboratory capacity to isolate $S$. Typhi from blood is absent or inadequate and the prevalence of resistant $S$. Typhi at both the individual and population level is unknown.

\section{Age distribution}

Two contemporary population-based studies address the age distribution of typhoid fever and suggest that, in areas highly endemic for typhoid fever, infection is more common in infants and pre-school children than in older persons $[14,15]$. The age distribution of typhoid fever has been modelled for typhoid fever at high (>100/100000 per year), medium (10-100/ 100000 per year), and low $(<10 / 100000$ per year) incidence countries using data from studies evaluating the age distribution of the infection $[14,15]$ and using surveillance data [7]. The resulting age distribution curves are reproduced in Figure 2 [1]. 
Table 4. Hospital-based studies of typhoid fever intestinal perforation, 1984-2005

\begin{tabular}{|c|c|c|c|c|c|c|c|c|c|}
\hline \multicolumn{3}{|l|}{ Location } & \multirow[b]{2}{*}{ HDI } & \multirow[b]{2}{*}{ Years } & \multirow{2}{*}{$\begin{array}{l}\text { Age } \\
\text { range } \\
(\mathrm{yr})\end{array}$} & \multirow{2}{*}{$\begin{array}{l}\text { No. of } \\
\text { illnesses }\end{array}$} & \multirow{2}{*}{$\begin{array}{l}\text { No. with } \\
\text { perforation }\end{array}$} & \multirow{2}{*}{$\begin{array}{l}\text { Perforation } \\
\text { rate }\end{array}$} & \multirow[b]{2}{*}{ Ref. } \\
\hline Area & Region & Country & & & & & & & \\
\hline Africa & S Africa & South Africa & Medium & 1993-95 & All & 102 & 5 & $4 \cdot 9$ & {$[10]$} \\
\hline Asia & E Asia & Taiwan & Medium & $1982-95$ & Children & 71 & 2 & $2 \cdot 8$ & [68] \\
\hline Asia & S Asia & Bangladesh & Medium & 1975-86 & All & 552 & 18 & $3 \cdot 3$ & [20] \\
\hline Asia & W Asia & Turkey & Medium & $1981-90$ & Children & 680 & 4 & $0 \cdot 6$ & [84] \\
\hline Oceania & Melanesia & $\begin{array}{l}\text { Papua New } \\
\text { Guinea }\end{array}$ & Medium & 1984-90 & All & 516 & 7 & $1 \cdot 4$ & [83] \\
\hline
\end{tabular}

HDI, Human development index.

Table 5. Hospital-based studies of typhoid fever mortality, 1984-2005

\begin{tabular}{|c|c|c|c|c|c|c|c|c|c|}
\hline \multicolumn{3}{|l|}{ Location } & \multirow[b]{2}{*}{ HDI } & \multirow[b]{2}{*}{ Year(s) } & \multirow{2}{*}{$\begin{array}{l}\text { Age } \\
\text { range } \\
(\mathrm{yr})\end{array}$} & \multirow{2}{*}{$\begin{array}{l}\text { No. of } \\
\text { illnesses }\end{array}$} & \multirow{2}{*}{$\begin{array}{l}\text { No. of } \\
\text { deaths }\end{array}$} & \multirow[b]{2}{*}{ CFR } & \multirow[b]{2}{*}{ Ref. } \\
\hline Area & Region & Country & & & & & & & \\
\hline Africa & W Africa & Gabon & Medium & $1992-96$ & All & 71 & 5 & $7 \cdot 0$ & [63] \\
\hline Africa & S Africa & South Africa & Medium & $1977-83$ & Children & 585 & 12 & $2 \cdot 1$ & [64] \\
\hline Africa & S Africa & South Africa & Medium & $1993-95$ & All & 102 & 1 & $1 \cdot 0$ & [10] \\
\hline Africa & S Africa & Zimbabwe & Low & 1984 & $\begin{array}{l}\text { Children } \\
<14\end{array}$ & 100 & 0 & 0 & [65] \\
\hline Asia & SE Asia & Thailand & Medium & 1986-2000 & Children & 19 & 0 & 0 & [66] \\
\hline Asia & SE Asia & Thailand & Medium & 1977-84 & Children & 163 & 0 & 0 & [67] \\
\hline Asia & E Asia & Taiwan & Medium & $1982-95$ & Children & 71 & 0 & 0 & [68] \\
\hline Asia & SE Asia & Indonesia & Medium & 1989-90 & $14-60$ & 105 & 5 & 5 & [13] \\
\hline Asia & SE Asia & Vietnam & Medium & 1993-94 & All & 293 & 9 & $0 \cdot 3$ & [69] \\
\hline Asia & SE Asia & Malaysia & Medium & $1981-90$ & $0-12$ & 46 & 0 & 0 & [70] \\
\hline Asia & SE Asia & Malaysia & Medium & $1984-6$ & Children & 137 & 2 & $1 \cdot 5$ & [71] \\
\hline Asia & SE Asia & Malaysia & Medium & 1975-9 & All & 121 & 2 & $1 \cdot 7$ & [72] \\
\hline Asia & SE Asia & Pakistan & Low & $1986-9$ & Children & 355 & 7 & $2 \cdot 0$ & [73] \\
\hline Asia & S Asia & Pakistan & Low & $1988-93$ & Children & 876 & 18 & $2 \cdot 1$ & [74] \\
\hline Asia & S Asia & Pakistan & Low & 1988-93 & Children & 1158 & 19 & $1 \cdot 6$ & [19] \\
\hline Asia & S Asia & Pakistan & Low & 1994 & All & 62 & 0 & 0 & [25] \\
\hline Asia & S Asia & Bangladesh & Medium & $1975-86$ & All & 552 & 24 & $4 \cdot 3$ & [20] \\
\hline Asia & S Asia & Bangladesh & Medium & $1980-81$ & All & 88 & 7 & $8 \cdot 0$ & [75] \\
\hline Asia & S Asia & Bangladesh & Medium & $1975-83$ & All & 323 & 18 & $5 \cdot 5$ & [8] \\
\hline Asia & SE Asia & Philippines & Medium & 1994 & All & 422 & 9 & $2 \cdot 1$ & [76] \\
\hline Asia & W Asia & Turkey & Medium & 1982-92 & Children & 27 & 4 & $14 \cdot 8$ & [77] \\
\hline Asia & S Asia & India & Medium & $1988-92$ & Children & 394 & 13 & $3 \cdot 3$ & [78] \\
\hline Asia & S Asia & India & Medium & 1990-92 & Children & 172 & 4 & $2 \cdot 3$ & [79] \\
\hline Asia & S Asia & India & Medium & 1990-91 & All & 102 & 1 & $1 \cdot 0$ & {$[80]$} \\
\hline Asia & S Asia & India & Medium & $1980 \mathrm{~s}$ & Adults & 125 & 11 & $8 \cdot 8$ & [81] \\
\hline Asia & S Asia & Pakistan & Low & 1986-89 & Children & 355 & 16 & $4 \cdot 5$ & [73] \\
\hline $\begin{array}{l}\text { Latin America } \\
\text { and Caribbean }\end{array}$ & Caribbean & Haiti & Low & 1989-91 & $\geqslant 14$ & 217 & 20 & $9 \cdot 2$ & {$[82]$} \\
\hline Oceania & Melanesia & $\begin{array}{l}\text { Papua New } \\
\text { Guinea }\end{array}$ & Medium & $1984-90$ & All & 374 & 52 & $13 \cdot 9$ & [83] \\
\hline
\end{tabular}

HDI, Human development index; CFR, case-fatality rate.

No population-based study has evaluated agespecific mortality for typhoid fever. Although by no means benign [15], typhoid fever is thought to be less severe in children [16-18] than in adults. Hospital inpatient series show that infants are at greater risk of dying from typhoid fever than older children $[19,20]$. 


\section{Geographic distribution}

The best summary of the geographic distribution of typhoid fever has been modelled from populationbased incidence studies (Table 2 and earlier studies), adjusted for age-specific incidence curves (Fig. 2), and diagnostic test sensitivity (Table 3 ). The resulting data on geographic distribution of typhoid fever are summarized in Figure 1 [1]. The very few contemporary studies from which this figure was developed are illustrated with open symbols $(\bigcirc)$. Based on these data sources, most typhoid fever illnesses occur among infants and young children in South and South East Asia.

\section{Temporal distribution}

The incidence of typhoid fever is reputed to follow seasonal patterns [21]. In some locations a peak occurs in the hot, dry months of the year. This is often attributed to a concentration of organisms in water when supply is inadequate due to lack of rain. However, in other places, a peak is reported during the rainy season. This is often attributed to a breakdown in the systems that separate sewage from drinking water. A review of typhoid fever seasonality publications between 1984 and 2005 confirms that both seasonal patterns are reported [22-25].

\section{Pathogen-specific preventive measures}

Three licensed vaccines are available to prevent typhoid fever; parenteral inactivated whole-cell vaccines, oral attenuated $S$. Typhi Ty21a vaccine, and parenteral Vi capsular polysaccharide vaccine. These vaccines confer about $70 \%$ protection in older children and adults and do not protect young children or infants [26-30]. More recently a conjugate vaccine has been developed that binds Vi to a non-toxic recombinant protein that is antigenically identical to Pseudomonas aeruginosa exotoxin A (Vi-rEPA). In a clinical trial in Vietnam, the vaccine showed $90 \%$ efficacy in children aged 2-5 years [31] and this level of protection was sustained for more than 3 years after vaccination [32]. To date, routine typhoid fever vaccination has rarely been adopted in typhoid-endemic countries [33]. Reasons include cost, lack of protection for young children, difficulties integrating currently available typhoid vaccines with current expanded programme on immunization (EPI) schedules, and the need for repeated booster doses.
Non-vaccine measures for typhoid fever prevention can be divided into community measures and individual measures [3]. Community measures for typhoid fever prevention include promotion of hand washing; sanitary disposal of human faeces; provision of safe drinking water; sanitary food preparation; pasteurization or boiling of milk and dairy products; quality control procedures for the food industry; and shellfish sanitation. Individual measures for typhoid fever prevention include education of patients, convalescents, and carriers in personal hygiene; breastfeeding throughout infancy; exclusion of typhoid carriers from food handling and from patient care provision; and provision of typhoid fever vaccine to persons at high risk due to occupation or travel.

Commonly recommended measures for typhoid fever control include reporting illnesses to health authorities, use of enteric precautions for ill patients until no fewer than three stool samples have proved negative for $S$. Typhi, safe disposal of faeces, urine, and soiled articles, identification and management of the source of infection, identification and screening of exposed individuals, and specific treatment of ill persons and carriers with an effective antimicrobial agent, most often a fluoroquinolone. Identification of chronic asymptomatic carriers through serological screening of the general population in an endemic area appears not to be useful [34]. Typhoid fever epidemics are managed by epidemiological identification of the source, disinfection of drinking water, followed by control measures directed to the epidemiologically identified source, e.g. intensive search for the case or carrier, selective elimination of suspected contaminated food, pasteurization or boiling of milk [3]. In a large waterborne epidemic of typhoid fever in Dushanbe, Tajikistan, implementation of chlorination of the city water supply lead to a dramatic reduction in typhoid fever incidence [12]. The introduction of widespread sewage treatment in Santiago, Chile, in 1992 was associated with a $90 \%$ reduction of typhoid fever and hepatitis A [35, 36]. Although currently not routinely recommended, one study shows that $\mathrm{Vi}$ vaccine deployed in a typhoid fever epidemic provided $71 \%$ protection against infection [37].

Case-control studies indicate that the predominant route of transmission of typhoid fever varies both in time and location. For example, in Tajikistan after independence from the Soviet Union deterioration of the municipal water treatment and distribution system led to lack of chlorination, equipment failure, and back-siphonage. This was associated with 
Table 6. Data gaps and research needs for typhoid fever in low- and medium-HDI countries

\begin{tabular}{|c|c|}
\hline Morbidity & $\begin{array}{l}\text { - Population-based surveillance for Salmonella enterica serotype Typhi incidence in } \\
\text { low HDI countries } \\
\text { - Population-based surveillance for Salmonella enterica serotype Typhi incidence in } \\
\text { sub-saharan Africa and from Central and South America }\end{array}$ \\
\hline Complications & $\begin{array}{l}\text { - Rates of complications in population-based studies of typhoid fever } \\
\text { - Evaluation of the effect of HIV co-infection on typhoid fever incidence and complications }\end{array}$ \\
\hline $\begin{array}{l}\text { Epidemic } v s . \text { endemic } \\
\text { disease }\end{array}$ & $\begin{array}{l}\text { - Assessment of contribution of outbreaks in the setting of large population-based } \\
\text { studies of typhoid fever incidence }\end{array}$ \\
\hline Mortality & $\begin{array}{l}\text { - Determination of case-fatality rates and mortality rates in adequately sized } \\
\text { population-based studies } \\
\text { - Identification of modifiable risk factors for death through cohort studies nested in } \\
\text { population-based disease incidence studies } \\
\text { - Intervention studies to ascertain the benefit of reducing mortality-associated risk factors } \\
\text { - Improved surveillance of antimicrobial resistance } \\
\text { - Treatment trials to evaluate antimicrobial agents for the treatment of drug-resistant } \\
\text { typhoid fever }\end{array}$ \\
\hline Age distribution & $\begin{array}{l}\text { - Surveillance among all age groups, with incidence reported for total population as well } \\
\text { as by age group }\end{array}$ \\
\hline Geographic distribution & $\begin{array}{l}\text { - Population-based incidence data from sub-saharan Africa and Central and } \\
\text { South America to strengthen regional disease incidence estimates } \\
\text { - Population-based incidence data from most low- and medium-human development } \\
\text { index countries to strengthen country level incidence estimates }\end{array}$ \\
\hline Seasonality & $\begin{array}{l}\text { - Collection of data and analysis regarding temporal distribution of typhoid fever over } \\
\text { multi-year periods, with reporting using strict case definitions that include culture } \\
\text { confirmation and contextual environmental information such as rainfall, flooding, } \\
\text { population density and degree of urbanization }\end{array}$ \\
\hline
\end{tabular}

HDI, Human development index.

widespread consumption of faecally contaminated water and a massive typhoid fever outbreak in 1997 [12]. In Pakistan, eating ice cream, eating food from a roadside cabin during the summer months, taking antimicrobials during the 2 weeks before illness onset, and drinking water at the worksite were independently associated with typhoid fever [38]. In Indonesia, recent typhoid fever in the household, not using soap for hand washing, sharing food from the same plate, and no toilet in the household were independent risk factors for typhoid fever [39]. The fraction of typhoid fever illness attributable to water, specific foods, or to typhoid fever carriers is poorly characterized both globally and at the local and regional levels. The lack of data on attribution limits the ability of policy-makers and public health officials to plan for transmission-based interventions to interrupt typhoid fever.

\section{Diagnostics}

The gold standard test for diagnosis of typhoid fever remains the culture of bone marrow. The sensitivity of this test exceeds $90 \%$ and the specificity is $100 \%$, assuming that bacterial identification methods are performed correctly [40]. Because obtaining samples for culture of bone marrow is invasive and requires technical expertise and equipment, blood culture is more often used for diagnosis even though the sensitivity of a single culture is about $40 \%$ [40-42]. Blood culture is more effected by prior antimicrobial use than bone marrow culture [42]. The sensitivity of blood culture for typhoid fever is improved by collection of larger volumes of blood [42].

The surface antigens of $S$. Typhi are shared by many other salmonellae and with other bacterial pathogens. This hampers specific serological 
diagnosis. Commercially available serological assays such as Multi-Test Dip-S-Ticks (PANBIO INDX Inc., Baltimore, MD, USA), TyphiDot (Malaysian Biodiagnostic Research SDN BHD, Singapore, Malaysia), and TUBEX (IDL Biotech, Sollentuna, Sweden) all have shortcomings of both sensitivity and specificity, with the Widal test performing the worst of all (Table 3).

\section{DISCUSSION}

This review highlights the large gaps in data on the burden of typhoid fever infections for low- and medium-HDI countries (Table 6). It also identifies additional research needs with respect to the burden of disease among infants and population-based rates of typhoid fever complications and mortality. Comprehensive microbiological, clinical, and population-based epidemiological studies using blood culture confirmation of cases or using novel study designs with alternative diagnostic methods could help to address these data gaps and further inform prevention. However, the implementation of such research would lead to and would require improvements in patient management that could be expected to reduce case fatality and complication rates.

Contemporary population-based studies using blood culture confirmation of cases are lacking or are extremely limited in all socioeconomic groups and geographic regions. Data are particularly limited for populations living in low-HDI countries and those in sub-Saharan Africa and Central and South America, where laboratory diagnostic capacity for typhoid fever may not be widely available. Of the 10 contemporary, population-based studies of typhoid fever that evaluate disease incidence using blood culture for confirmation of cases the reported incidence ranges from 13 to 976/100 000 persons per year. These studies are likely to have been done preferentially in highincidence sites that make generalization of results from a single location to a whole country or region difficult. Only two studies report typhoid fever incidence simultaneously in different age groups making the burden among infants and young children difficult to estimate $[14,15]$. Preliminary evidence that the age distribution of typhoid fever varies according to disease incidence needs to be confirmed by population-based studies conducted in both low and medium typhoid fever incidence settings. A sound understanding of both incidence and mortality in different age groups is needed to make decisions about typhoid fever vaccine policy. Although large typhoid fever epidemics are recognized to occur occasionally [12], the relative contribution of epidemic $v s$. endemic diseases to the overall burden of typhoid fever has not been established.

Little is known about long-term temporal trends in typhoid fever incidence in low- and medium-HDI countries and what underlies such trends. To improve understanding of long-term trends, collection of data and analysis is required over multi-year periods, with reporting using strict case definitions that include culture confirmation and contextual environmental information such as rainfall, flooding, population density and degree of urbanization.

No population-based typhoid fever study in our series reported rates of typhoid fever complications. Consequently, we calculated rates from hospitalbased studies. Hospital-based typhoid fever studies showed median (range) intestinal perforation rates of $2 \cdot 8 \%(0 \cdot 6-4 \cdot 9 \%)$. Rates of complications other than intestinal perforation are not consistently reported in contemporary hospital-based studies. Therefore, the total morbidity attributable to typhoid fever cannot currently be calculated. It is probable that hospitalbased studies capture only the most severe illness among persons who have access to health care and therefore such studies may overestimate the rates of complications [7]. No study has systematically evaluated the effect of HIV co-infection on typhoid fever incidence and complications. Data are needed on the rates of complications from population-based studies. Such tracking might be done in areas where a discrete number of health facilities provide care for the majority of the population.

With the continued emergence of antimicrobial resistance in $S$. Typhi [43], improved surveillance for antimicrobial resistance is needed as well as continued efforts to evaluate antimicrobial agents for the treatment of drug-resistant $S$. Typhi infection.

Only five population-based studies in our series reported data that allowed calculation of the typhoid fever CFR. In these five studies, the median (range) typhoid fever CFR was $0 \%(0-1 \cdot 8 \%)$. When using case studies to examine rates of typhoid fever complications, it is likely that the ethics of populationbased typhoid fever incidence or vaccine studies will require enhanced clinical management of patients which could alter the measured CFR leading to underestimation of the CFR in such studies.

Policy-makers lack data to make decisions about pathogen-specific prevention measures for typhoid 
fever. The effect of non-vaccine prevention and control measures, such as implementation of safe drinking water, has not been systematically evaluated. The fraction of typhoid fever attributable to various routes of transmission (i.e. water, food) has not been established. Furthermore, cost-benefit assessments, such as a comparison of the rapid implementation of water disinfection with the introduction of typhoid vaccines in the epidemic setting, have not been done. Finally, studies confirming the beneficial effect and feasibility of scaling up both vaccine interventions and non-vaccine measures in intervention studies would be useful to inform decisions on the distribution of resources to specific prevention programmes.

The diagnosis of typhoid fever provides an ongoing challenge both for patient care and for surveillance. The diagnosis of typhoid fever can rarely be made using clinical history and physical examination alone [7]. Bone marrow aspiration and culture is the goldstandard diagnostic test [40], but it is not practical for use in the field. Consequently, blood culture has become the practical gold standard, yet it lacks sensitivity and is also challenging to implement in the field in resource-poor settings. While a number of rapid diagnostic tests have been evaluated, none achieves the sensitivity of bone marrow culture and none has the specificity of the culture-based methods [44]. Continued efforts are needed to identify a simple, rapid diagnostic test for typhoid fever. In parallel, efforts should be made to expand access to and to improve blood culture technologies in low- and medium-HDI countries.

There are substantial gaps in the current understanding of typhoid fever epidemiology. These gaps are so large that policy-makers may have insufficient information to select the most appropriate interventions to reduce typhoid fever illnesses and death and to monitor the effects of such interventions. To address gaps in the current understanding of typhoid fever incidence, complications, and CFR, large population-based studies using blood culture confirmation of cases are needed in representative sites, especially in low-HDI countries outside Asia. Although such information is expensive to acquire, it would be invaluable for the prioritization of precious public health resources for pathogen-specific interventions, such as vaccination programmes targeting typhoid fever. Additional data regarding complications, modifiable risk factors for mortality, the burden of disease among infants, effectiveness of non-vaccine prevention measures, and improved diagnostics would add greatly to our knowledge of typhoid fever.

\section{ACKNOWLEDGEMENTS}

This work was supported in part by the U.S. National Institutes of Health Fogarty International Center and by grant number 32143 from the Bill and Melinda Gates Foundation 'Assessment of diarrhea disease burden and public health programs to control diarrhea in Asian subcontinent and Africa'.

\section{DECLARATION OF INTEREST}

None.

\section{REFERENCES}

1. Crump JA, Luby SP, Mintz ED. The global burden of typhoid fever. Bulletin of the World Health Organisation 2004; 82: 346-353.

2. Mandell GL, Bennett JE, Dolin R. Principles and Practice of Infectious Diseases, 6th edn. New York, NY: Elsevier/Churchill Livingstone, 2005.

3. Heymann DL. Control of Communicable Diseases Manual, 18th edn. Washington, DC: American Public Health Association, 2004.

4. Reller ME, et al. Sexual transmission of typhoid fever: a multistate outbreak among men who have sex with men. Clinical Infectious Diseases 2003; 37: 141-144.

5. Hornick RB, Woodward TE. Appraisal of typhoid vaccine in experimentally infected human subjects. Transactions of the American Clinical and Climatological Association 1967; 78: 70-78.

6. Murray PR, et al. Manual of Clinical Microbiology, 8th edn. Washington, DC: ASM Press, 2003, pp. 2310.

7. Crump JA, et al. Estimating the incidence of typhoid fever and other febrile illnesses in developing countries. Emerging Infectious Diseases 2003; 9: $539-544$.

8. Butler T, et al. Typhoid fever complicated by intestinal perforation: a persisting fatal disease requiring surgical management. Reviews of Infectious Diseases 1985; 7: 244-256.

9. Hosoglu S, et al. Risk factors for enteric perforation in patients with typhoid fever. American Journal of Epidemiology 2004; 160: 46-50.

10. Khan M, et al. Influence of sex on clinical features, laboratory findings, and complications of typhoid fever. American Journal of Tropical Medicine and Hygiene $1999 ; 61: 41-46$.

11. Huang DB, DuPont HL. Problem pathogens: extraintestinal complications of Salmonella enterica serotype Typhi infection. Lancet Infectious Diseases 2005; 5: 341-348. 
12. Mermin JH, et al. A massive outbreak of multidrugresistant typhoid fever in Tajikistan associated with consumption of municipal water. Journal of Infectious Diseases 1999; 179: 1416-1422.

13. van den Bergh ETAM, et al. Outcome in three groups of patients with typhoid fever in Indonesia between 1948 and 1990. Tropical Medicine and International Health 1999; 4: 211-215.

14. Sinha A, et al. Typhoid fever in children aged less than 5 years. Lancet 1999; 354: 734-737.

15. Brooks WA, et al. Bacteremic typhoid fever in an urban slum, Bangladesh. Emerging Infectious Diseases 2005; 11: 326-329.

16. Ferreccio $\mathbf{C}$, et al. Benign bacteremia caused by Salmonella Typhi and Paratypi in children aged younger than 2 years. Journal of Pediatrics 1984; 104: 899-900.

17. Mulligan TO. Typhoid fever in young children. British Medical Journal 1971; 4: 665-667.

18. Arora RK, et al. Multidrug resistant typhoid fever: study of an outbreak in Calcutta. Indian Pediatrics 1992; 29: 61-66.

19. Bhutta ZA. Impact of age and drug resistance on mortality in typhoid fever. Archives of Diseases in Childhood 1996; 75: 214-217.

20. Butler T, et al. Patterns of morbidity and mortality in typhoid fever dependent on age and gender: review of 552 hospitalized patients with diarrhea. Reviews of Infectious Diseases 1991; 13: 85-90.

21. Hunter GW, Strickland GT, Magill AJ. Hunter's Tropical Medicine and Emerging Infectious Diseases, 8th edn. Philadelphia: W.B. Saunders Company, 2000, pp. 1192.

22. Battikhi MN. Occurence of Salmonella Typhi and Salmonella Paratyphi in Jordan. New Microbiology 2003; 26: 363-373.

23. Saha SK, et al. Typhoid fever in Bangladesh: implications for vaccination policy. Pediatric Infectious Diseases 2001; 20: 521-524.

24. Lin F-YC, et al. The epidemiology of typhoid fever in the Dong Thap Province, Mekong Delta region of Vietnam. American Journal of Tropical Medicine and Hygiene 2000; 62: 644-648.

25. Mirza SH, Beeching NJ, Hart CA. The prevalence and clinical features of multi-drug resistant Salmonella Typhi infections in Baluchistan, Pakistan. Annals of Tropical Medicine and Parasitology 1995; 89: 515-519.

26. Ivanoff B, Levine MM, Lambert PH. Vaccination against typhoid fever: present status. Bulletin of the World Health Organisation 1994; 72: 957-971.

27. Acharya IL, et al. Prevention of typhoid fever in Nepal with the Vi capsular polysaccharide of Salmonella Typhi. New England Journal of Medicine 1987; 317: 1101-1104.

28. Klugman KP, et al. Protective activity of Vi capsular polysaccharide vaccine against typhoid fever. Lancet 1987; 2: 1165-1169.

29. Levine MM, et al. Duration of efficacy of Ty21a, attenuated Salmonella Typhi live oral vaccine. Vaccine 1999; 17 (Suppl. 2): S22-27.
30. Centers for Disease Control and Prevention. Typhoid immunization: recommendations of the Advisory Committee on Immunization Practices (ACIP). Morbidity and Mortality Weekly Reports 1994; 43: 1-8.

31. Lin FY, et al. The efficacy of a Salmonella Typhi Vi conjugate vaccine in two-to-five-year-old children. New England Journal of Medicine 2001; 344: 1263 1269.

32. Lanh MN, et al. Persistent efficacy of Vi conjugate vaccine against typhoid fever in young children. New England Journal of Medicine 2003; 349: 1390-1391.

33. Bodhidatta L, et al. Control of typhoid fever in Bangkok, Thailand, by annual immunization of schoolchildren with parenteral typhoid vaccine. Reviews of Infectious Diseases 1987; 9: 841-845.

34. Gupta A, et al. Evaluation of community-based serologic screening for identification of chronic Salmonella Typhi carriers in Vietnam. International Journal of Infectious Diseases 2006; 10: 309-314.

35. Cabello F, Springer AD. Typhoid fever in Chile 1977-1990: an emergent disease. Revista Medica de Chile 1997; 125: 474-882.

36. Wolff M. Changes in the epidemiology of infectious diseases in Chile from 1990 to 2000. Revista Medica de Chile 2002; 130: 353-362.

37. Yang HH, et al. Efficacy trial of $\mathrm{Vi}$ polysaccharide vaccine against typhoid fever in south-western China. Bulletin of the World Health Organisation 2001; 79: 625-631.

38. Luby SP, et al. Risk factors for typhoid fever in an endemic setting, Karachi, Pakistan. Epidemiology and Infection 1998; 120: 129-138.

39. Vollaard AM, et al. Risk factors for typhoid and paratyphoid fever in Jakarta, Indonesia. Journal of the American Medical Association 2004; 291: 2607-2615.

40. Gilman RH, et al. Relative efficacy of blood, urine, rectal swab, bone-marrow, and rose-spot cultures for recovery of Salmonella Typhi in typhoid fever. Lancet 1975; 1: 1211-1213.

41. Hoffman SL, et al. Bone marrow aspirate culture superior to streptokinase clot culture and $8 \mathrm{~mL} 1: 10$ blood-to-broth ratio blood culture for diagnosis of typhoid fever. American Journal of Tropical Medicine and Hygiene 1986; 35: 836-839.

42. Wain J, et al. Quantitation of bacteria in bone marrow from patients with typhoid fever: relationship between counts and clinical features. Journal of Clinical Microbiology 2001; 39: 1571-1576.

43. Crump JA, et al. Reevaluating fluoroquinolone breakpoints for Salmonella enterica serotype Typhi and for non-Typhi salmonellae. Clinical Infectious Diseases 2003; 37: 75-81.

44. Olsen SJ, et al. Evaluation of rapid diagnostic tests for typhoid fever. Journal of Clinical Microbiology 2004; 42: $1885-1889$.

45. Srikantiah P, et al. Population-based surveillance of typhoid fever in Egypt. American Journal of Tropical Medicine and Hygiene 2006; 74: 114-119.

46. Klugman KP, et al. Immunogenicity, efficacy, and serological correlate of protection of Salmonella Typhi Vi 
capsular polysaccharide vaccine three years after immunization. Vaccine 1996; 14: 435-438.

47. Simanjuntak $\mathbf{C H}$, et al. Oral immunisation against typhoid fever in Indonesia with Ty21a vaccine. Lancet 1991; 338: 1055-1059.

48. Levine MM, et al. Large-scale field trial of Ty21a live oral typhoid vaccine in enteric-coated capsule formulation. Lancet $1987 ; 1$ : 1049-1052.

49. Levine MM, et al. Comparison of enteric-coated capsules and liquid formulation of Ty21a typhoid vaccine in randomised controlled field trial. Lancet 1990; 336: 891-894.

50. Black RE, et al. Efficacy of one or two doses of Ty21a Salmonella Typhi vaccine in enteric-coated capsules in a controlled field trial. Vaccine 1990; 8: 81-84.

51. Wahdan MH, et al. Controlled field trial of a typhoid vaccine prepared with a nonmotile mutant of Salmonella Typhi Ty2. Bulletin of the World Health Organisation 1975 ; 52: 69-73.

52. Wahdan MH, et al. A controlled field trial of live Salmonella Typhi strain Ty 21a oral vaccine against typhoid: three-year results. Journal of Infectious Diseases 1982; 145: 292-295.

53. Chuttani CS, et al. Controlled field trial of high-dose oral killed typhoid vaccine in India. Bulletin of the World Health Organisation 1977; 55: 643-644.

54. Polish Typhoid Committee. Evaluation of typhoid vaccines in the laboratory and in a controlled field trial in Poland. Bull WHO 1965; 32: 15-27.

55. Hejfec LB, et al. A controlled field trial and laboratory study of five typhoid vaccines in the USSR. Bulletin of the World Health Organisation 1966; 34: 321339.

56. Hejfec LB. Results of the study of typhoid vaccines in four controlled field trials in the USSR. Bulletin of the World Health Organisation 1965; 32: 1-14.

57. Hejfec LB, et al. Controlled field trials of paratyphoid $B$ vaccine and evaluation of the effectiveness of a single administration of typhoid vaccine. Bulletin of the World Health Organisation 1968; 38: 907-915.

58. Hejfec LB, et al. A controlled field trial to evaluate the protective capacity of a single dose of acetone-killed agar-grown and heat-killed broth-grown typhoid vaccines. Bulletin of the World Health Organisation 1969; 40: 903-907.

59. Yugoslav Typhoid Commission. A controlled field trial of the effectiveness of acetone-dried and inactivated and heat-phenol-inactivated typhoid vaccines in Yugoslavia. Bulletin of the World Health Organisation 1964; 30: 623-630.

60. Yugoslav Typhoid Commission. A controlled field trial of the effectiveness of phenol and alcohol typhoid vaccines. Bulletin of the World Health Organisation 1962; 26: 357-369.

61. Ashcroft MT, et al. A seven-year field trial of two typhoid vaccines in Guyana. Lancet 1967; 2: 10561059.

62. Tapa S, Cvjetanovic B. Controlled field trial on the effectiveness of one and two doses of acetone-inactivated and dried typhoid vaccine. Bulletin of the World Health Organisation 1975; 52: 75-80.

63. Okome-Nkoumou M, et al. Typhoid and paratyphoid fever in adults in the internal medicine department at Libreville, Gabon. Sante 2000; 10: 205-209.

64. Ellis ME, Moosa A, Hillier V. A review of typhoid fever in South African black children. Postgraduate Medical Journal 1990; 66: 1032-1036.

65. Topley JM. Mild typhoid fever. Archives of Diseases in Childhood 1986; 61 : 164-167.

66. Wongsawat J, Pancharoen C, Thisyakorn U. Typhoid fever in children: experience in King Chulalongkorn Memorial Hospital. Journal of the Medical Association of Thailand 2002; 85: 1247-1250.

67. Thisyakorn U, Mansuwan P, Taylor DN. Typhoid and paratyphoid fever in 192 hospitalized children in Thailand. American Journal of Diseases of Childhood 1987; 141: 862-865.

68. Chiu C-H, et al. Typhoid fever in children: a fourteenyear experience. Acta Paediatrica Taiwan 2000; 41 : 28-32.

69. Hoa NTT, et al. Community-acquired septicaemia in southern Viet Nam: the importance of multidrugresistant Salmonella Typhi. Transactions of the Royal Society of Tropical Medicine and Hygiene 1998; 92: 503-508.

70. Yap YF, Puthucheary SD. Typhoid fever in children: a retrospective study of 54 cases from Malaysia. Singapore Medical Journal 1998; 39: 260-262.

71. Choo KE, et al. Typhoid fever in hospitalized children in Kelantan, Malaysia. Annals of Tropical Paediatrics 1988; 8: 207-212.

72. Brown GW, et al. Febrile illness in Malaysia: an analysis of 1,629 hospitalized patients. American Journal of Tropical Medicine and Hygiene 1984; 33: 311-315.

73. Bhutta ZA, et al. Multidrug-resistant typhoid in children: presentation and clinical features. Review of Infectious Diseases 1991; 13: 832-836.

74. Bhutta ZA. Therapeutic aspects of typhoidal salmonellosis in childhood: the Karachi experience. Annals of Tropical Paediatrics 1996; 16: 299-306.

75. Islam SS, Shahid NS. Morbidity and mortality in a diarrhoeal diseases hospital in Bangladesh. Transactions of the Royal Society of Tropical Medicine and Hygiene 1986; 80: 748-752.

76. Abucejo PE, et al. Blood culture confirmed typhoid fever in a provincial hospital in the Philippines. Southeast Asian Journal of Tropical Medicine and Public Health 2001; 3: 531-536.

77. Secmeer G, et al. Salmonella Typhi infections. Turkish Journal of Pediatrics 1995; 37: 339-344.

78. Biswal N, et al. Enteric fever: a changing perspective. Indian Pediatrics 1994; 31: 813-819.

79. Rasaily R, et al. Multi-drug resistant typhoid fever in hospitalized children: clinical, bacteriological and epidemiological profiles. European Journal of Epidemiology 1994; 10: 41-46.

80. Rao PS, et al. Emergence of multidrug-resistant Salmonella Typhi in rural southern India. American 
Journal of Tropical Medicine and Hygiene 1993; 48: 108-111.

81. Gupta SP, et al. Current clinical patterns of typhoid fever: a prospective study. Journal of Tropical Medicine and Hygiene 1985; 88: 377-381.

82. Olle-Goig JE, Ruiz L. Typhoid fever in rural Haiti. Bulletin PAHO 1993; 27 : 382-388.
83. Richens J. Typhoid fever in the highlands of Papua New Guinea 1984-1990: a hospital-based perspective. PNG Medical Journal 1995; 38: 305314.

84. Kizilcan F, et al. Complications of typhoid fever requiring laparotomy during childhood. Journal of Pediatric Surgery 1993; 28: 1490-1493. 